Meta

Journal des traducteurs

Translators' Journal

\title{
L'enseignement de la traduction à Montréal
}

\section{Paul A. Horguelin}

Volume 11, numéro 4, décembre 1966

La formation du traducteur

URI : https://id.erudit.org/iderudit/003116ar

DOI : https://doi.org/10.7202/003116ar

Aller au sommaire du numéro

Éditeur(s)

Les Presses de l'Université de Montréal

ISSN

0026-0452 (imprimé)

1492-1421 (numérique)

Découvrir la revue

Citer cet article

Horguelin, P. A. (1966). L'enseignement de la traduction à Montréal. Meta,

11(4), 145-146. https://doi.org/10.7202/003116ar d'utilisation que vous pouvez consulter en ligne.

https://apropos.erudit.org/fr/usagers/politique-dutilisation/ 
ENSEIGNEMENT DE LA TRADUCTION À MONTRÉAL

\section{INSTITUT DE TRADUCTION}

L'Institut de traduction, qui célébrait l'an dernier le $25^{\mathrm{e}}$ anniversaire de sa fondation, est rattaché à l'Université de Montréal depuis la rentrée d'octobre 1965. Sa direction administrative relève de l'Extension de l'enseignement, et les cours sont donnés avec la collaboration pédagogique du Département de linguistique et de langues modernes de la Faculté des lettres.

Le changement de régime a été suivi d'une profonde réforme des structures et de l'enseignement. Pour assurer la liaison entre les deux départements intéressés, d'une part, et les professeurs et étudiants, d'autre part, l'Université a nommé un directeur pédagogique et une directrice administrative. Elle a fait établir ensuite un plan directeur qui sert de cadre aux réformes en cours. Ces réformes, qui portent notamment sur les normes et les programmes, s'échelonneront sur une période de deux ans, à compter de cette année. Le nouveau programme sera donc entièrement en vigueur à la rentrée de 1967.

Les principaux objectifs du plan directeur sont de relever le niveau des cours et d'assurer une meilleure coordination de l'enseignement. Pour parvenir à ces fins, plusieurs moyens pratiques seront mis en œuvre, de l'examen d'entrée à la classe terminale. L'examen d'admission - qui portait jusqu'ici sur un thème et une version - est remplacé par un test permettant de vérifier les connaissances de l'élève dans les quatre domaines suivants: langue française, langue anglaise, traduction, culture générale. Selon les résultats du test, les candidats sont dirigés vers des cours de rattrapage en anglais ou en français (cours dispensés par l'Extension de l'enseignement et ne relevant pas de l'Institut), ou bien admis en préparatoire ou en première année. Par ce moyen, on évite d'encombrer les classes d'éléments qui n'ont pas une formation suffisante pour profiter des cours de traduction proprement dits, mais aussi de rejeter systématiquement des éléments « récupérables 》.

La classe préparatoire s'adresse aux élèves qui ont une bonne connaissance de l'anglais et du français, mais qui ne sont pas prémunis contre les écueils de la 
traduction sur le plan lexical: anglicismes, faux amis, calques, etc. Le programme du cours comporte donc un aspect négatif: perte des mauvaises habitudes linguistiques, et un aspect positif: utilisation du dictionnaire et enrichissement du vocabulaire. À la fin de l'année, les élèves sont pour la plupart prêts à traduire et ils peuvent alors commencer le cycle normal d'études qui comprend trois années.

Les changements apportés au programme des cours du cycle normal visent principalement à introduire un certain degré de spécialisation par année et à assurer l'uniformité et la continuité des cours d'une classe à l'autre. C'est ainsi que la première année sera consacrée plus spécialement à la traduction commerciale; la deuxième année, à la traduction technique et spécialisée; la troisième année, à la traduction littéraire et publicitaire. Cette spécialisation, qui est d'ailleurs une orientation plutôt qu'un cadre rigide, a l'avantage de permettre aux professeurs d'étudier au cours de l'année les grands principes d'une forme de traduction (on sait que le processus de la traduction technique, par exemple, n'est pas le même que celui de la traduction littéraire); comme autres avantages, on pourrait ajouter une meilleure utilisation des aptitudes des professeurs - l'expérience professionnelle étant importante dans l'enseignement de la traduction -, un regroupement de la bibliographie et des sources de documentation, la possibilité pour l'élève de se tracer un programme suivi de lecture. Le deuxième but visé est la gradation et la coordination de l'enseignement. Il est en effet essentiel que les élèves constatent qu'ils progressent d'une façon ordonnée, selon un programme bien défini, et que tous arrivent à l'examen de fin d'études avec un bagage comparable. On s'est donc efforcé d'établir une gradation dans la difficulté des textes et des matières, chaque professeur ayant en outre une indication des normes à atteindre. Parallèlement, l'enseignement de la stylistique comparée (selon la méthode et les manuels de MM. Vinay et Darbelnet) a été réparti sur les trois années du cycle, et cette matière figure au programme des examens. Selon le plan directeur, la norme en fin d'études «correspond aux connaissances et à l'aptitude que doit posséder le candidat à un poste de traducteur. En d'autres termes, le diplômé doit faire honneur à l'Institut et à la profession. 》

[CI À la rentrée de 1965, l'Institut comptait 386 élèves répartis de la façon suivante: 61 en préparatoire ( 2 classes), 147 en première ( 6 classes), 89 en deuxième ( 3 classes), 70 en troisième ( 2 classes) et 19 en quatrième (cours de perfectionnement). Les inscriptions pour l'année scolaire 1966-1967 dépasseront certainement ce chiffre, car les cours de traduction jouissent d'une faveur accrue depuis quelques années.

Au seuil d'une nouvelle étape de sa carrière, l'Institut de traduction envisage donc l'avenir avec confiance et détermination. Enrichi des enseignements du passé, mais aussi résolu à suivre l'évolution des idées et des techniques, il poursuit sa tâche qui est de former des traducteurs compétents et conscients de leurs responsabilités.

Paul A. Horguelin Montréal 